\title{
Betreuung von Frühgeborenen und Neugeborenen mit Erkrankungen von Schwangerschaft bis Wochenbett
}

\author{
Oda von Rahden
}

\begin{abstract}
Auch in den Wochen nach einer Frühgeburt sind Eltern mit ihrem Kind vielfach noch unsicher. Deshalb ist es im frühen Wochenbett bedeutsam, die Eltern-Kind-Bindung zu fördern. In Teil 2 der Serie vermittelt die Autorin, wie Sie Familien nach der Frühgeburt und Klinikentlassung adäquat begleiten. Lesen Sie, wie es mit den in Teil 1 vorgestellten Familien weiterging, und finden Sie in den Kommentaren zu den Fallbeispielen Handlungsempfehlungen für die Praxis.
\end{abstract}

\section{Die ersten Tage und Wochen nach der Geburt}

\section{Fallbeispiel: Lena* \& Felix*}

Lena* (alle mit * markierten Namen sind redaktionell verändert) wird am Tag nach der Geburt von einer Hebamme auf der Wochenbettstation erklärt, wie und wie oft sie Milch abpumpen soll. Felix* hat sich für den Rest der Woche krankschreiben lassen und kommt morgens in die Klinik. Lena* wartet schon sehr auf ihn, denn sie möchte nicht ohne ihn zu den Kindern auf die Neo gehen. Heute Morgen hat sie dort bereits angerufen und nach Laura* und Sophie* gefragt. Die Kinder seien „soweit stabil“ wurde ihr gesagt. Felix* schiebt Lena* im Rollstuhl zur Neo. Mit einem ängstlichen Gefühl betreten sie die Station. Nun wird ihnen gezeigt, wo sie sich die Hände waschen sollen und wie man sich die Hände desinfiziert. Danach bringt eine Kinderkrankenschwester beide in einen Raum mit vielen Inkubatoren. Sie zeigt ihnen, wo Laura* und Sophie* stehen. Lena* nimmt heute zum ersten Mal die ganzen Monitore, Infusionsgeräte und Geräusche wahr. Ständig scheint etwas zu piepen. Die Kinderkrankenschwester zeigt, wie man die Klappen am Inkubator öffnet und wie sie die Babys anfassen können. Nun erst erkennt Lena*, wie zart und klein ihre Babys sind, und sie muss weinen. Die Kinderkrankenschwester nimmt Lenas* Hände und legt sie auf Laura*. Lena* kann Lauras* Wärme und ihre Bewegungen spüren. Felix* fasst

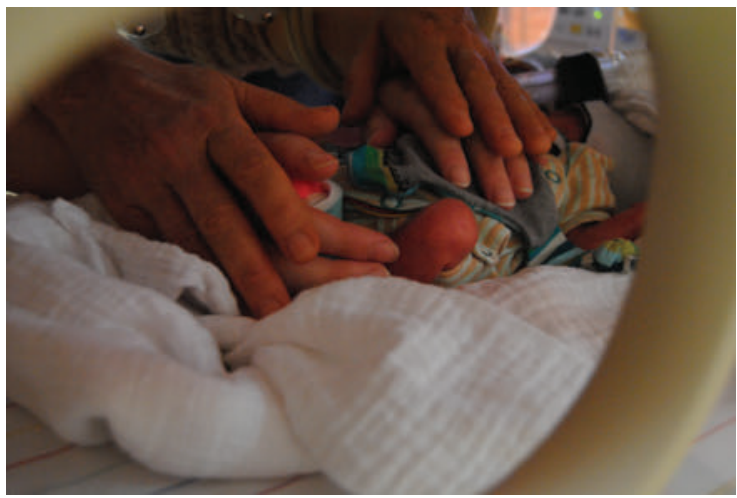

Abb. 1 Bindungsfördernde Pflege bei einem 3 Wochen alten Frühgeborenen. (Foto: v. Rahden [rerif])

Sophies* Händchen an. Nach einer Stunde merkt Lena*, dass ihre Sectionaht schmerzt. Trotzdem fühlt sie sich etwas besser. Das Sehen und Spüren der Kinder gibt ihr etwas Zuversicht.

\section{Fallbeispiel: Johanna* \& Samuel*}

Johanna* hat die erste Nacht nach der Geburt sehr unruhig geschlafen. Sie macht sich Gedanken, wie die nächsten Wochen werden sollen. Samuel* ist noch in der Probezeit und kann keinen Urlaub nehmen. Sie weiß nicht, wie sie sich zugleich um Charlotte*, ihre dreijährige Tochter, und Robert* in der Klinik kümmern soll. Charlotte* war noch nie lange von ihrer Mutter getrennt 
und Johanna* macht sich Sorgen, dass sie unter der neuen Situation leiden wird. Sie geht in den ersten Tagen immer wieder zu Robert* und darf ihn nach zwei Tagen bereits känguruen. Sie genießt diese Zeit und döst manchmal ein wenig dabei. Nach anfänglichen Hemmungen lernt sie schnell, wie sie ihn im Inkubator selbst wickeln kann und fühlt sich im Umgang mit ihm nach wenigen Tagen bereits relativ sicher. Sie pumpt Muttermilch ab, wie es ihr von der Hebamme der Klinik gezeigt wurde. Weiterhin rät ihr die Hebamme, für die Zeit zu Hause eine nachbetreuende Hebamme zu suchen. Lena* ruft einige Hebammen an, bekommt aber meist Absagen oder kann nur Nachrichten auf Anrufbeantwortern hinterlassen. Da sie bei Charlotte* keine Probleme mit dem Stillen hatte, beschießt sie, es erst einmal alleine zu probieren.

Nach 6 Tagen wird sie aus der Klinik entlassen. Von nun an ist ihr Tagesablauf straff organisiert: Morgens um 6 Uhr Milch abpumpen, anschließend Hausarbeit, Frühstück und Charlotte* zum Kindergarten bringen. Danach fährt sie gleich weiter in die Klinik. Wenn sie Glück hat, ist sie rechtzeitig für die 9-Uhr-Versorgung da. Wenn die Zeit reicht, pumpt sie in der Klinik wieder Muttermilch $a b$, wickelt Robert* und känguruht anschließend mit ihm. Nach knapp 3 Stunden wird Robert* wieder in den Inkubator gelegt, denn Lena* muss wieder zum Kindergarten und Charlotte* abholen. Am Nachmittag beschäftigt sich Lena* mit Charlotte*, versucht nebenbei etwas im Haushalt zu tun und pumpt noch einmal Milch ab. Am späten Nachmittag kommt Samuel* nach Hause. Nun fährt einer von beiden wieder in die Klinik zu Robert*, der andere versorgt Charlotte* und bringt sie ins Bett. Abends vor dem Schlafengehen pumpt Lena* ein letztes Mal ab und stellt fast jeden Tag fest, dass sie nicht oft genug abgepumpt hat.

\section{Diskussion des Fallbeispiels}

Den Wundheilungs- und Rückbildungsprozessen wird seitens der Eltern in Anbetracht der gesundheitlichen



- Abb. 2 Die Mutter bekommt ihr 4 Tage altes Frühgeborenes zum Känguruhen. (Foto: privat [rerif])
Problematik ihres Kindes wenig Aufmerksamkeit beigemessen. Aufgrund der fehlenden Schonung und psychischen Belastung treten Komplikationen bei der Milchbildung und Wundheilungsstörungen häufiger auf und werden oft später erkannt. Der Hebamme kommt hier, im Sinne der Sekundärprävention, eine Schlüsselfunktion zu. Sie überwacht die Wundheilungs- sowie Rückbildungsvorgänge der Wöchnerin und kann weiterhin darauf hinwirken, dass - bei allen Sorgen und Ängsten um das frühgeborene Kind - der Regenerationsbedürftigkeit der Wöchnerin angemessen Rechnung getragen wird. So lässt sich das Risiko von Komplikationen minimieren.

Viele Frühgeboreneltern stehen nach der Entlassung der Mutter aus der Klinik zudem vor der Frage, wie sie die Entfernung zwischen Heimatort und Perinatalzentrum bewältigen sollen. Einige Kliniken bieten Elternzimmer an oder haben ein Elternhaus in Kliniknähe. Wenn keine weiteren Kinder zu Hause zu versorgen sind oder andere Verpflichtungen eine Präsenz zu Hause erfordern (z. B. Landwirtschaft), erleichtert die kliniknahe Unterbringung diese frühe Familienphase erheblich.

Viele Frühgeborenenmütter sind zum Geburtszeitpunkt noch nicht in Hebammenbetreuung und wissen nicht, dass ihnen diese zusteht. Andere haben bereits eine Hebamme, müssen sich aber nun, aufgrund der großen Entfernung zum Heimatort, eine neue suchen. Das Klinikpersonal steht in der Pflicht, Eltern über ihren Anspruch auf Wochenbettbetreuung zu informieren und möglichst bei der Suche zu helfen. Hebammen sollten der Wochenbettbetreuung nach Frühgeburt genügend Gewicht beimessen, auch wenn „nur“ die Wöchnerin, nicht aber das Kind zu betreuen ist.

\section{Fallbeispiel: Lena* \& Felix*}

Laura* und Sophie* haben die ersten Lebenstage gut überstanden, die Ärzte berichten den Eltern, die Kinder seien stabil. Am dritten Lebenstag konnten beide Kinder extubiert werden und haben nun eine CPAP-Maske. Am 4. Lebenstag dürfen Lena* und Felix* mit den Kindern känguruhen. Wegen der vielen Kabel und Atemhilfe haben sie anfänglich Angst, als man die Kinder aus dem schützenden Inkubator nimmt. Aber nachdem beide eine Weile das Kind auf der Brust spüren, fühlen sie sich sicherer und genießen es sehr.

Lena* wird nach einer Woche aus der Klinik entlassen und zieht in ein Zimmer im benachbarten Elternhaus. Sie hat eine Hebamme gefunden, die sie so kurzfristig noch betreuen kann. Felix* schläft werktags zu Hause und geht zur Arbeit. An den Wochenenden ist er bei Lena* und den Kindern. Am 10. Lebenstag geht es Laura* nicht gut. Lena* fällt auf, dass sie blass aussieht und sehr unruhig ist. Auch die Pflegekräfte und Ärzte machen sich Sorgen. Es wird bei Laura* Blut abgenommen und 
festgestellt, dass sie eine Infektion hat. Eine Röntgenuntersuchung ergibt, dass sich ihr Darm entzündet hat. Sie darf nun nicht mehr zum Känguruhen aus dem Inkubator und wird auch sonst möglichst wenig bewegt. Lena* fühlt sich sehr hilflos, denn sie kann Laura* nun nur noch durch die Inkubatorklappe berühren und mit ihr sprechen.

Gegen Abend verschlechtert sich ihr Zustand so sehr, dass Laura* wieder intubiert werden muss und die Ärzte beschließen, sie zu operieren. Im OP-Aufklärungsgespräch erfahren Lena* und Felix*, dass Lauras* Zustand sehr kritisch ist und man nicht wisse, ob sie die OP überstehen wird. Es wird angeboten, eine Nottaufe durchzuführen, wodurch den beiden der Ernst der Lage noch deutlicher wird. So schnell können sie das nicht entscheiden. Der Eingriff dauert 2 Stunden. Lena* und Felix* kommt es wie eine Ewigkeit vor. Nach der OP wird ihnen erklärt, dass man ein Stück Darm entfernen musste und dass Laura* nun einen künstlichen Darmausgang hat. Laura* darf vorerst keine Muttermilch bekommen, erst in den nächsten Tagen wird langsam mit dem Nahrungsaufbau begonnen. Man müsse hoffen, dass alles gut verheilt.

Für Lena* ist es nach diesem Ereignis wieder sehr schwer, auf die Neo zu gehen. Sie fürchtet sehr, dass man ihr von Komplikationen berichten könnte. Deshalb ruft sie morgens erst auf der Station an und erkundigt sich, wie die Nacht war, bevor sie selbst dort hingeht. Mit Felix* steht sie mehrmals am Tag per SMS im Kontakt, abends telefonieren sie. Nach Lauras* OP geht Lenas* Muttermilch zurück. Obwohl Lena* über den Tag verteilt 7- bis 8-mal abpumpt, wird es weniger. Lena* bespricht dies mit ihrer Hebamme. Diese erklärt Lena*, dass das eine Folge der psychischen Belastung sein könne. Sie rät Lena*, am Inkubator abzupumpen, also unmittelbar bei den Kindern. Weiterhin findet die Hebamme, Lena* solle sich zwischen den Versorgungszeiten der Kinder häufiger ausruhen. Lena* und Felix* beschließen, dass Felix* eine Woche frei nimmt und im Elternhaus bleibt. Lena* kann nun morgens länger liegen bleiben und legt sich auch nachmittags noch mal hin, während Felix* bei den Kindern ist.

\section{Diskussion des Fallbeispiels}

Durch regelmäßige Besuche gewinnt die Hebamme Einblick in das Familienleben und das soziale Netz der Familie und kann somit frühzeitig erkennen, welche unterstützenden Faktoren (Ressourcen), aber auch welche Risikofaktoren den Wochenbettverlauf, neben den Belastungen aufgrund der Frühgeburt, beeinflussen.

Neben der Traumatisierung durch die Frühgeburt kann es, je nachdem wie die gesundheitliche Entwicklung des Kindes ist, im Verlauf zu weiteren Belastungen oder ReTraumatisierungen kommen. Das Erlebte kann in aller
Regel erst viel später verarbeitet werden. Verwandte und Freunde können die Situation der Frühgeboreneneltern oft kaum nachvollziehen. Aufgrund des geburtshilflichen Fachwissens sind Hebammen gute Gesprächspartnerinnen für die noch unerfahrenen Frühgeborenenmütter. Durch ihr offenes Ohr für die Schilderungen des Erlebten, der Sorgen und Ängste, legen sie einen Grundstein für eine spätere emotionale Verarbeitung des Geschehens.

\section{Fallbeispiel: Johanna* \& Samuel*}

Robert* entwickelt sich in den nächsten Tagen gut. Bereits in seiner 2. Lebenswoche kann er ohne CPAP atmen und trinkt mitunter aus der Flasche. Beim Känguruen legt Johanna* Robert* ab und zu an und Robert* beginnt langsam, kleine Mengen Muttermilch aus der Brust zu trinken. Johanna* fährt nun abends, obwohl sie erschöpft ist, wieder in die Klinik, damit sie Robert* anlegen kann. Nach 16 Tagen wacht Johanna* morgens mit Kopfschmerzen auf. Sie fühlt sich krank und stellt fest, dass sie Fieber hat. Samuel ${ }^{*}$ meldet sich bei der Arbeit ab, bringt Charlotte* in den Kindergarten und fährt anschließend mit Johanna* zum Gynäkologen. Dort wird festgestellt, dass Johanna* einen Lochialstau und eine Endometritis hat. Sie wird in die Klinik eingewiesen und dort antibiotisch behandelt. Johanna* ist fühlt sich völlig ausgelaugt und schläft viel. Beim Abpumpen kommt kaum noch Milch. 3 Tage kann sie nicht zu Robert*. Obwohl sie weiß, dass Samuel* jeden Tag bei Robert* ist und mit ihm kängurut, leidet sie sehr unter der Trennung.

\section{Diskussion der Fallbeispiele}

Die Fallbeispiele zeigen, dass der Wochenbettverlauf maßgeblich durch die Hebammenbetreuung beeinflusst werden kann. Eine telefonische Beratung, wie viele Hebammen es anbieten, reicht hierfür nicht. Die Hausbesuche dienen, neben der üblichen Überwachung von Wundheilung und Rückbildung, der Beratung bei der erschwerten Milchbildung. Aufgrund der verkürzten Schwangerschaft hatten viele noch gar keine Gelegenheit, sich mit dem Thema Stillen zu befassen, so dass den Wöchnerinnen häufig grundlegende Informationen zu Milchbildung, Milchspendereflex und Anlegetechniken fehlen. Die Wöchnerin benötigt deshalb deutlich mehr Informationen und weiterhin eine individuelle Beratung in Bezug auf die Laktation. Während des stationären Aufenthalts wird den Wöchnerinnen meist nur Grundlegendes zur Bedienung der Milchpumpe, Hygiene und Pumphäufigkeit erklärt. Die anfängliche Empfehlung, 8mal täglich zu pumpen lässt sich für viele nicht realisieren, oder lässt sich nur sehr schwer in den Tagesablauf integrieren. Eine allgemeine gehaltene Beratung hilft hier wenig. Die Hebamme sollte, nach Beurteilung der Brust und des Milchflusses, individuell beraten, wie sinnvollerweise abzupumpen ist. Sie kennt die aktuellen Umstände der Wohnsituation oder etwaiger anderer 
Belastungen der Wöchnerin und kann dies berücksichtigen. Ihre Empfehlungen sind somit für die Wöchnerin besser umsetzbar und es wird ein unbefriedigendes Abpumpen vermieden.

Die Hebamme hat, aufgrund ihrer Einblicke in die Lebenssituation der Familie, die Möglichkeit, die Wöchnerin zu unterstützen, einen strukturierten Tagesablauf zu entwickeln, der genügend Raum für den Bindungsaufbau zum Kind lässt und gleichermaßen ausreichend Regenerationsphasen enthält. Nach der Entlassung aus der Klinik, sind Hebammen häufig die einzige medizinische Fachkraft, die regelmäßigen Kontakt zu der Wöchnerin und ihrer Familie hat. Daher kommt ihr eine zentrale Rolle bei der Früherkennung einer Wochenbettdepression zu (s. Teil 1 der Serie [4]). Zur Behandlung von psychischen Störungen im Wochenbett muss die Hebamme die Wöchnerin in adäquate Behandlung überleiten. Eine Kenntnis der regionalen Versorgungsangebote bei psychischer Erkrankung im Wochenbett ist hierzu erforderlich.

\section{Klinikentlassung des Kindes und Eingewöhnung im häuslichen Umfeld}

Der Unterstützungsbedarf durch die Hebamme bleibt nicht auf die Dauer des stationären Aufenthalts des Kindes beschränkt. Die Entlassung des Kindes findet Wochen, mitunter erst Monate nach der Geburt statt. Das Wochenbett der Mutter ist möglicherweise bereits abgeschlossen.

\section{Fallbeispiel Lena* \& Felix*}

Laura* und Sophie* haben sich in den weiteren Wochen gut entwickelt. Lena* ist es gelungen, die Muttermilchmenge zu steigern, so dass sie, 8 Wochen nach der Geburt, die Kinder endlich an die Brust legen kann. Anfänglich lecken Laura* und Sophie* wenige Tropfen Muttermilch, dann beginnt Laura* ein wenig zu saugen. Mit Unterstützung einer Still-und Laktationsberaterin

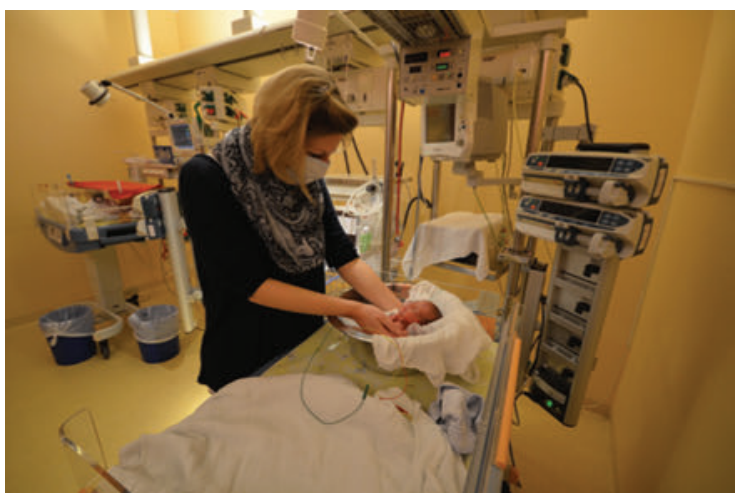

Abb. 3 Die Mutter badet ihr 4 Wochen altes Frühchgeborenes. (Foto: privat [rerif]) gelingt es schließlich, dass beide Kinder jede zweite Malzeit gestillt werden und die anderen Malzeiten aus der Flasche trinken. Lena* pumpt nach wie vor 4- bis 5-mal täglich ab. Seit sie die Kinder nach Bedarf stillt, ist die Zeit noch knapper geworden.

Lena* und Felix* haben mit der Zeit ihre Töchter gut kennen gelernt. Sie wissen, dass Laura* quengelig wird, wenn sie nach dem Stillen zu schnell auf den Bauch gelegt wird und dass Sophie* am liebsten auf dem Bauch liegt. Sie haben auch gelernt, Laura*s künstlichen Darmausgang zu versorgen und können den Beutel selbst wechseln. Bis bei Laura* der Anus praeter zurück verlegt werden kann, dauert es noch einige Wochen, so dass sie einige Zeit auch zu Hause damit zurechtkommen müssen.

Felix* hat an den Abenden, wenn er zu Hause war, das Kinderzimmer hergerichtet und Lena* war 2-mal zu Hause und hat die Babykleidung gewaschen und eingeräumt. Nun ist alles fertig für die Entlassung. Lena* und Felix* freuen sich sehr darauf, endlich als Familie nach Hause zu gehen. Zugleich machen sie sich Sorgen, ob sie all dem gewachsen sein werden, was auf sie zukommt.

Nach der Entlassung des Frühgeborenes aus der Klinik, werden die Eltern in vielfacher Hinsicht gefordert. Die Familie muss in der neuen Konstellation zusammenwachsen und es muss ein Alltag, möglicherweise mit einem pflegebedürftigen Kind, neu etabliert werden. Trotz bindungsfördernder Pflege und Eltern-Kind-Zimmern in der Klinik, fühlen sich Eltern nach langem Klinikaufenthalt im Umgang mit ihrem Kind in der Regel noch unsicher. Die Entlassung aus der Klinik sollte daher gut geplant sein und individuelle Problemlagen und das persönliche Erleben der Eltern berücksichtigen [1].

\section{Fallbeispiel: Johanna* \& Samuel*}

Johanna* konnte nach 4 Tagen wieder aus der Klinik entlassen werden. Samuel* ist inzwischen von der Arbeit freigestellt, sodass Johanna* und Samuel* sich aufteilen können, wer bei Robert* in der Klinik ist und wer Charlotte* betreut und sich um den Haushalt kümmert. Johanna* hat während der Tage in der Klinik nur noch selten Muttermilch abgepumpt, sodass schließlich kaum noch Milch da war. Das Pumpen war für sie dadurch sehr frustrierend. Letztlich hat sie dann abgestillt. Robert* ist inzwischen sehr gewachsen, kann ohne Unterstützung atmen und hält seine Körpertemperatur selber. Er saugt gerne und trinkt gut aus der Flasche, sodass ihm nach nur wenigen Tagen der Umstellung die Magensonde gezogen werden konnte. Robert* nicht stillen zu können, bedauert Johanna* sehr. Sie weiß um die Vorteile der Muttermilch und hat ein schlechtes Gewissen, dass Robert* nun Säuglingsnahrung bekommt. Sie besucht ihn jeden Tag in der Klinik und versorgt inn dort selbstständig. Manchmal ist Robert* sehr unruhig und lässt sich nur schwer beruhigen. 
Die Kinderkrankenschwestern erklären Johanna* dann, Robert* habe zu viel Luft geschluckt und müsse bäuern oder dass ihn Blähungen plagen. Bei Charlotte* wusste Johanna* immer, was ihr fehlt. Dass sie sich bei ihrem zweiten Kind auf einmal so unselbstständig fühlt, frustriert sie. Daher ruft Johanna* die Hebamme wieder mal an, die sie bei Charlotte* betreut hatte, um nach der Entlassung eine Ansprechpartnerin zu haben.

\section{Diskussion der Fallbeispiele}

Wie auch bei reif und gesund geborenen Kindern begleitet die Hebamme Familien nicht nur in medizinsicherer, sondern auch in psychosozialer Hinsicht. Erst nach der Entlassung des Frühgeborenen aus der Klinik kann die Familie sich in der neuen Konstellation einleben. Es gilt, eine neue Tagesstruktur zu entwickeln, die dem besonderen Bedarf des Frühgeborenen entspricht, aber gleichermaßen an die Alltagsnotwendigkeiten und Bedürfnisse der restlichen Familienmitglieder angepasst ist. Dies kann ein langwieriger Prozess sein, zumal davon auszugehen ist, dass alle Familienmitglieder nach den Belastungen der vergangenen Wochen und Monate sehr regenerationsbedürftig und daher wenig belastbar sind.

Indem die Hebamme die Belange der Familie im Blick hat, kann sie diesen Prozess begleiten. Sie weiß, ob und in welchem Umfang ein soziales Netz die Eltern unterstützt, und sie kennt etwaige andere Risikofaktoren, die den Familienstart zu Hause erschweren. Somit kann sie erkennen, ob weitere professionelle Unterstützung erforderlich ist oder ob längerfristige Betreuung, z. B. durch eine Familienhebamme, sinnvoll ist.

\section{Handlungsempfehlungen für die Betreuung nach der Klinikentlassung}

Der späte Betreuungszeitpunkt nach Entlassung des Kindes bedeutet, dass in medizinischer Hinsicht weniger die körperlichen Belange der Mutter im Fokus der Betreuung stehen als vielmehr die Überwachung der Entwicklung des ehemaligen Frühgeborenen. Das Gedeihen des Kindes muss durch regelmäßige Gewichtskontrollen geprüft werden. Bei Frühgeborenen, die mit einer speziellen Frühgeborenen- oder Anfangsnahrung ernährt werden, lässt sich die Trinkmenge problemlos kontrollieren. Bei mit Muttermilch ernährten Frühgeborenen stellt sich dies schwieriger dar. Es kommt häufig vor, dass ein Kind zum Zeitpunkt seiner Entlassung noch nicht die gesamte benötigte Nahrungsmenge aus der Brust trinkt und zusätzlich mit der Flasche gefüttert wird. Für die Mutter bedeutet dies, dass sie weiterhin Muttermilch abpumpen muss. Es empfiehlt sich, neben dem 
Stillen, ungefähr 4-mal täglich abzupumpen, bis das Kind 3500 Gramm wiegt. 14 Tage nach der Entlassung kann meist begonnen werden, das Abpumpen zu reduzieren.

Das genaue Vorgehen sollte nach Beurteilung der Gesamtsituation von der Hebamme individuell angeleitet werden. Langfristig sollte immer das Ziel sein, dass das ehemalige Frühgeborene voll gestillt wird und auf das Pumpen verzichtet werden kann. Für die Mütter ist das ein schwieriger Weg. Da sie in den ersten Lebenswochen ihres Kindes über jedes Gramm Gewichtszunahme froh waren, fällt es ihnen nun schwer, das Kind nur noch nach Bedarf zu stillen, keine Wiegeproben mehr zu machen und darauf zu vertrauen, dass das Baby von sich aus so viel trinkt, wie es braucht. Die Stillberatung stellt daher einen zentralen Aspekt der Nachbetreuung nach Entlassung des Frühgeborenen dar und erfordert gute Fachkenntnisse der Hebamme.

Ein Großteil der Frühgeborenen oder chronisch kranken Neugeborenen wird mit einer Medikation entlassen. Neben Vitamin D wird werden häufig Eisentropfen und Vitamin A verordnet, gelegentlich auch Diuretika oder Coffein. Frühgeborene, die unter Coffeintherapie entlassen wurden, müssen weiterhin mit einem Monitor überwacht werden. Frühgeborene mit einer bronchopulmonalen Dysplasie benötigen über die Dauer der stationären Behandlung hinaus eine Inhalationstherapie und gelegentlich auch noch Sauerstoff. Die korrekte Medikation sollte den Eltern in der Klinik vermittelt werden. Dennoch muss die nachbetreuende Hebamme prüfen, ob es den Eltern gelingt, die Medikamente korrekt zu verabreichen, oder ob eine Hilfestellung erforderlich ist.

Bei vielen Frühgeborenen sind, neben den regulären Kinderarztvorsorgen, weitere Kontrollen bei verschiedenen Fachärzten wie z. Bsp. Augenarzt oder Kardiologe erforderlich oder sie benötigen Physiotherapie. Auch bei der Koordination der medizinischen Termine kann die Hebamme die Eltern unterstützen, da dies manche Eltern überfordert. Eine vollständige Koordination der Kontrolltermine übersteigt jedoch unter Umständen ihren Aufgabenbereich. Dann sollte sie zur Nachbetreuung eine weitere Fachkraft ins Boot holen. So wird auch nach dem stationären Aufenthalt ein multiprofessionelles Team aktiv.

Für Familien, bei denen sich eine derart umfangreiche ambulante Nachbehandlung abzeichnet, sollten sinnvollerweise bereits vor der Entlassung aus der Klinik ein Betreuungskonzept erstellt und die Nachbetreuung mit allen beteiligten Berufsgruppen organisiert werden. Neben der Hebamme zählen hierzu eine sozialmedizinische Nachsorge und in manchen Fällen ein Pflegedienst. Bei größeren psychosozialen Problemlagen kann es auch sinnvoll sein, eine längerfristige Betreuung durch eine Familienhebamme zu initiieren.

\section{STICHWORT \\ Sozialmedizinische Nachsorge}

Nach der Entlassung des Frühgeborenen müssen Eltern je nach Gesundheitszustand des Kindes und psychosozialer Problemlage der Familie viele Herausforderungen im Alltag, möglicherweise mit einem pflegebedürftigen Kind, bewältigen. In diesen Fällen ist neben der Hebammenbetreuung eine zusätzliche Begleitung der Eltern im Rahmen der sozialmedizinischen Nachsorge sinnvoll. Diese Betreuung orientiert sich an der Handlungsmethode des Case

Managements und wird über die gesetzliche Krankenversicherung finanziert.

Die sozialmedizinische Nachsorge wird in einem Umfang von bis zu 20 Stunden, verteilt über einen Zeitraum von 6 bis 12 Wochen verordnet. Die Fachkräfte, die die Familie hierbei durch Hausbesuche betreuen sind, je nach Betreuungsschwerpunkt Kinderkrankenschwestern oder Sozialpädagoginnen, die eine Case-Management-Weiterbildung absolviert haben. Sozialmedizinische Nachsorge wird bundesweit von Nachsorgeeinrichtungen, z. B. Bunter Kreis e. V., angeboten. In den Teams sind Kinderkrankenschwestern, Sozialpädagoginnen, Psychologin und Kinderärztin vertreten [3].

Da sie den Übergang zwischen stationärer und ambulanter Versorgung erleichtern soll, kann sozialmedizinische Nachsorge nur im Anschluss an einen stationären Aufenthalt verordnet werden. Daher muss die Planung und Verordnung bereits Rahmen der Entlassungsplanung aus der Klinik erfolgen. Eltern empfinden es als zusätzliche Sicherheit, zu wissen, an wen sie sich bei etwaigen Unsicherheiten nach dem Klinikaufenthalt wenden können. Für die nachbetreuende Hebamme ergibt sich der Vorteil, dass sie mit anderen Fachkräften Rücksprache halten kann, die mit ihr die Familie weiterbetreuen. Andererseits ist die Hebamme gefordert, mit den anderen Fachpersonen zu kooperieren und Absprachen zu Betreuungsinhalten zu treffen.

\section{Fazit}

Bei einer Frühgeburt hatte die Mutter in den meisten Fällen noch keine Gelegenheit, sich eine betreuende Hebamme für das Wochenbett zu suchen. Dementsprechend sind derartige Anfragen bei den Hebammen meist sehr kurzfristig und erfordern eine zeitnahe Übernahme einer nicht vorgeplanten Wochenbettbetreuung. Es kann bei der Betreuung grob zwischen zwei Zeitfenstern unterschieden werden: Die Entlassung der Wöchnerin und, meist einige Wochen später, die Entlassung des Frühgeborenen. Beide 
Phasen haben aus medizinischer Sicht unterschiedliche Schwerpunkte. Neben den jeweiligen medizinischen Aspekten muss die Hebamme aber auch in starkem Maße psychosoziale Faktoren bei der Betreuung berücksichtigen. Grundsätzlich gilt, dass Wochenbettbesuche bei stationär behandeltem Kind nicht zwangsläufig in reduzierter Form stattfinden sollten. Die zeitliche Eingebundenheit der Eltern in der Kinderklinik erfordert hingegen von den Hebammen Organisationsgeschick und eine Flexibilität bei den Terminvereinbarungen.

Der Bedarf und Umfang der Betreuung hängt nicht in erster Linie von der medizinischen Brisanz der Frühgeburt oder kindlichen Erkrankung ab. Vielmehr ist das Zusammenwirken individueller familiärer Ressourcen und Risikofaktoren ausschlaggebend. Nur durch regelmäßigen persönlichen Kontakt in Form von Hausbesuchen ist eine Einschätzung des Bedarfs durch die nachbetreuende Hebamme möglich.

Der Förderung der Eltern-Kind-Bindung kommt, auch Wochen nach der Frühgeburt, ein großer Stellenwert zu, da viele Eltern im Umgang mit ihrem Kind noch unsicher sind. Eine gelungene Bindung stellt eine wichtige Maßnahme zur Prävention von Folgeproblemen und Kindesmisshandlungen dar. Denn die eingeschränkten Interaktionsmöglichkeiten der ersten Wochen können ein starkes Kontrollbedürfnis und die Entstehung unrealistischer Ängste bei Eltern zur Folge haben. Dies kann in einen überbehütenden Erziehungsstil münden [2] oder dazu führen, dass Eltern die kindlichen Bedürfnisse nicht erkennen. Werden Eltern von Anfang an im Kontakt mit ihrem Kind unterstützt, kann trotz zu früher Geburt eine gute Bindungsbasis geschaffen werden, sodass bereits im Alter von 6 Monaten kein Unterschied mehr im Bindungsverhalten zwischen frühund reif geborenen Kindern feststellbar ist [2].

Der fachkundigen und empathischen Begleitung der Hebamme kommt hierbei eine zentrale Bedeutung zu. Ihr Betreuungsangebot steht (im Gegensatz z. B. zur sozialmedizinischen Nachsorge) allen Wöchnerinnen zu, unabhängig davon, ob besondere medizinische Indikationen vorliegen. Die Hebammenbetreuung lässt der Familie eine professionelle Unterstützung zuteilwerden, die entscheidend zu einem gesunden Start ins Familienleben beiträgt und somit diverse Folgekomplikationen verhindern kann.

\section{Autorinnen / Autoren}

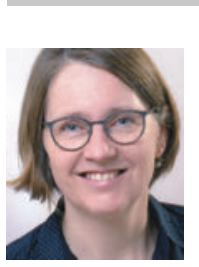

Dr. Oda von Rahden ist Hebamme und Dipl.Psychologin am Klinikum Oldenburg, Universitätsklinik für Kinder- und Jugendmedizin Elisabeth-Kinderkrankenhaus. Die Hilfen für Familien mit Frühgeborenen zählen zu ihren Arbeitsschwerpunkten.

\section{Korrespondenzadresse}

\author{
Klinikum Oldenburg \\ Universitätsklinik für Kinder- und Jugendmedizin - \\ Elisabeth-Kinderkrankenhaus \\ Dr. Oda von Rahden \\ Rahel-Straus-Straße 10 \\ 26133 Oldenburg \\ E-Mail: vonrahden.oda@klinikum-oldenburg.de
}

\section{Literatur}

[1] Gross-Letzelter M, Baumgartner M, Frühchen-Eltern Kovacic A. Eine sozialpädiatrische Studie. Lengerich: Papst; 2010

[2] Korja R, Latva R, Lehtonen L. The effects of preterm birth on mother-infant interaction and attachment during the infant's first two years. Acta Obstet Gynecol Scand 2012; 91(2).164173

[3] Podeswik A, Kanth E, Schreiber-Gollwitzer B, Labouvie H, Baur W, Otto A, Kusch M. Praxishandbuch pädiatrische Nachsorge. Modell Bunter Kreis. Augsburg: beta-Institutsverlag; 2007

[4] von Rahden O. Betreuung von Frühgeborenen und Neugeborenen mit Erkrankungen von Schwangerschaft bis Wochenbett. Die Hebamme 2019; 32: 12-21

\section{Bibliografie}

DOI https://doi.org/10.1055/a-0953-4205

Die Hebamme 2019; 32: 57-63

(c) Georg Thieme Verlag KG Stuttgart · New York

ISSN 0932-8122 